\title{
Multi-target Tracking in the Moving Background Based on Many Layer Wavelet Son-image Matching Method
}

\author{
Liu Gang ${ }^{1, a}$, Liu Bing ${ }^{1, b}$ \\ 1.Base Department, Aviation University of Air Force, Changchun, China, 130022 \\ amyrhlg@163.com, ${ }^{\mathrm{b}} 438317769 @ q q . c o m$
}

\begin{abstract}
Keywords:wavelet transform, moving targets tracking, block matching, figure center calculation Abstract. For the image shot by the CCD camera fixed on the moving carrier (such as plane or ship), there is the whole image movement by the movement of carrier besides the movement of targets. For detecting moving targets in the moving background and tracking them, an arithmetic based on wavelet transform multi-layer block matching tracking is suggested in this article. The wavelet of bior1.3 is used to decompose image. It is anti-symmetric wavelet and is fit for detecting variation information in horizontal and vertical direction. It can use many layer son-image to match and the vector of the whole background can be estimated. It can detect moving targets from the background by difference arithmetic, and can work out the figure center of target, and trace out the movement contrail of the targets. Compared with traditional block matching tracking arithmetic, it overcome some disadvantages, such as a mass of operation, inaccurate matching in the low SNR image. Otherwise the matching in $\mathrm{N} \times \mathrm{N}$ area in son-image is equal to the matching in $2^{\mathrm{n}} \mathrm{N} \times 2^{\mathrm{n}} \mathrm{N}$ area in oral image. The searching speed is faster than before. The experiment is done while the moving vector of two neighbor frame is less then 10 pixel and the moving vector of the moving targets in background is less then 5 pixel. The availability and feasibility of this arithmetic is proved by the result.
\end{abstract}

\section{Introduction}

When detecting moving targets in image serial, frequently image sensor is fixed on the moving carrier, such as infrared CCD camera fixed on a plane or a missile. In this way, the backgrounds of serial image are moving. For checking out the moving targets in image serial, the movement of the image sensor is approximately flat movement, So the whole movement vector of background is estimated by block matching method.

The merit of block matching method is better tracking ability, and easy realization. The whole searching method is in the common use, matching process is easy, it is easy to carry out by hardware, performance is all right, but the vast quality of operation is block to use. For conquering trouble, based on relativity between son-images, an arithmetic based on wavelet transform multi-layer block matching tracking is suggested. Operation quantity of moving estimate is reduced, the searching time is decreased.

\section{Acquiring of Multi-scale Decomposition of Discrete Image}

The wavelet of bior1.3 is used to decompose image. It is anti-symmetric wavelet and is fit for detecting variational information in horizontal and vertical direction.

The original image is decomposed by two layer wavelet, as shown in figure 1. Channel 1 is low frequency image of two layer decomposition. Channel 2 4 is high frequency image of two 
layer decomposition. Channel 5 7 is high frequency image of one layer decomposition. The high frequency noise energy mostly concentrate in the channel $5 \sim 7$, so these image isn' $t$ used to segment targets and background. A majority of low frequency energy concentrate in the channel 1.

\section{Block-matching search algorithm}

Using local full search in search algorithm, if within $29 \times 29$ range (suppose the background motion vector size of the two adjacent frames is no more than 20 pixels, and no more than 10 pixels in the low-frequency component of wavelet figure), with a $9 \times 9$ template matching. The MSE rule is the matching rule:

$$
C(x, y)=\frac{1}{N N} \sum_{i=1}^{N} \sum_{j=1}^{N}\left(Z_{1}(i, j)-Z_{0}(x+i, y+j)\right)^{2}
$$

$\mathrm{N} \times \mathrm{N}$ represents the pixel numbers of the participate matching operations, (x,y) represents an offset occurs in the $\mathrm{x} 、 \mathrm{y}$ direction, $\mathrm{Z} 1$ represents a motion vector block need to be estimated in the current frame, $\mathrm{ZO}$ is a candidate block that is within the search window in the reference frame. Obtaining the MSE C (x, y) of the pixels corresponded by Z1, Z0, obtained the smallest $\mathrm{C}(\mathrm{x}, \mathrm{y})$, its corresponding $(\mathrm{x}, \mathrm{y})$ is the current frame motion vector relative to the reference frame.

\section{Computation of Multi-target Figure Center}

After segment targets and background, we can give targets pixel a value of 255, other region 0. If we want to gain the trajectory of moving targets, or forecast the next position of targets, the figure center of targets must be made out.

It is supposed that the size of targets have been known. A search window of targets can be confirmed. We used $9 \times 9$ size of search window to search in the whole image. When nonzero pixel is searched, which is regarded as the center of the search window. The figure center of target can be assured as follow:

$$
X(n)=\frac{\sum X_{i} B(i, j)}{\sum B(i, j)} \quad, \quad Y(n)=\frac{\sum y_{i} B(i, j)}{\sum B(i, j)}
$$

Where $\mathrm{B}(\mathrm{i}, \mathrm{j})$ is gray-degree value of every pixel in the search window, $\mathrm{xi}$, yi is coordinate of corresponding every pixel. The $\mathrm{B}(\mathrm{i}, \mathrm{j})$ of every moving targets is 255 , the $\mathrm{B}(\mathrm{i}, \mathrm{j})$ of other pixel is 0 . $\mathrm{X}(\mathrm{n})$ and $\mathrm{Y}(\mathrm{n})$ is coordinate of targets figure center. If we compute the figure center like formula (2), one or two, even more figure center can be made out. Because the size of targets is possibly bigger than search window, the search window contain different part of the target, so that we can make out different value according to same target.

In this way we must classify horizontal and vertical coordinate by likeness degree. The like horizontal and vertical coordinate is classified as one target. Average value can be made out, as the figure center of one target.

\section{The Experiment Results}

As shown in Fig. 1, these are the frames of the Original Images. The center of the White-box is the moving targets that be tracked, the background motion vector of the adjacent two frames is less than 10 pixels, the motion vector of the moving target relative to the background is less than 5 pixels. We do the simulation experiment on Matlab6 by using the hierarchical block tracking 
target match algorithm based on wavelet transform, First of all, we do the two-dimensional discrete wavelet decomposition on the two frames with the adjacent motion image sequence number, and do the rough match between the two adjacent frames with the low frequency sub-image. The second, we do the exact-match on the Original Images. After determining the background Offset, we do the difference operation for the two adjacent images, and process the Domain value, then the moving target can be Segmented, we can calculate the background offset relative to the first frame. Then by translating the moving targets, the Tracks of the moving targets can be drawn, as shown in the Fig. 2. The coordinate values of the detected target' $\mathrm{s}$ first 10 frames are shown in the Table 1.

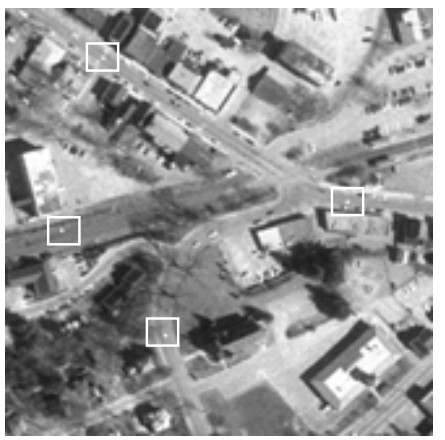

(a)The 1th frame

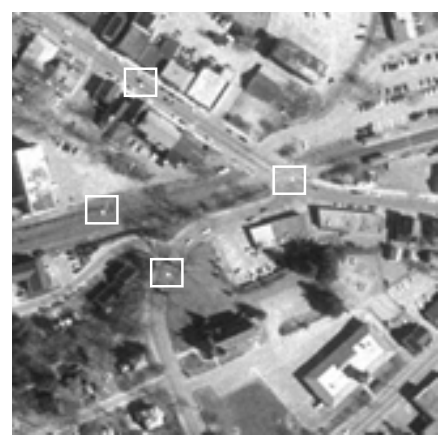

(b)The 22th Frame

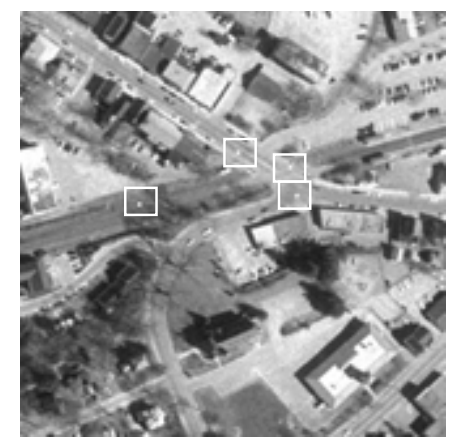

(c)The 58th Frame

Fig. 1 Original Image and the Positions of the Moving Targets

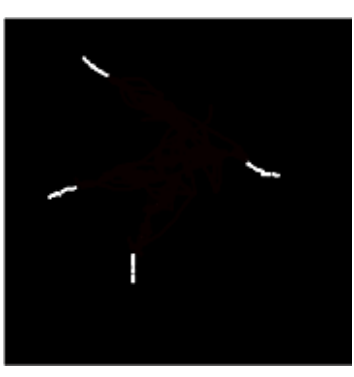

(a) The Trail after 12 Frames

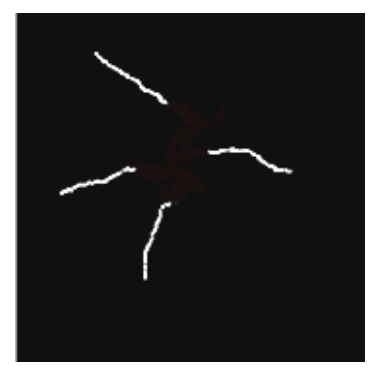

(b) The Trail after 33 Frames

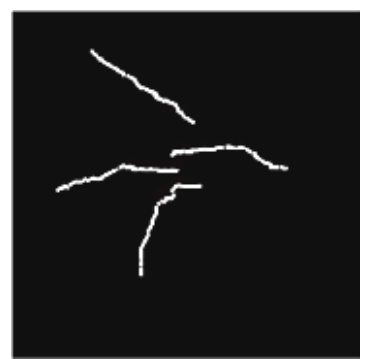

(c) The Trail after 54 Frames

Fig. 2 the Tracks of the Moving Targets in 12Frames, 33 Frames and 54 Frames

Table 1 Coordinate value of targets in first 10 frames (Unit: pixel)

\begin{tabular}{|c|c|c|c|c|c|c|c|c|}
\hline Targets No. & \multicolumn{2}{|c|}{1} & \multicolumn{2}{|c|}{2} & \multicolumn{2}{|c|}{3} & \multicolumn{2}{|c|}{4} \\
\hline Frames No. & Line & Column & Line & Column & Line & Column & Line & Column \\
\hline 1 & 40 & 68.5 & 111 & 174.5 & 125.5 & 48.5 & 175.5 & 87.5 \\
\hline 2 & 41 & 70.5 & 110 & 178 & 125.5 & 50 & 173.5 & 90 \\
\hline 3 & 43 & 72.5 & 110 & 181.5 & 126 & 51.5 & 172.5 & 92.5 \\
\hline 4 & 44.5 & 74 & 108.5 & 175.5 & 125.5 & 50 & 169.5 & 93.5 \\
\hline 5 & 46 & 75.5 & 107 & 172 & 125 & 52.5 & 166.5 & 94.5 \\
\hline 6 & 47 & 78.5 & 106 & 174.5 & 124 & 55.5 & 164 & 96.5 \\
\hline 7 & 48 & 79 & 105 & 171 & 124 & 56 & 162 & 97 \\
\hline 8 & 48.5 & 80.5 & 103 & 168.5 & 123.5 & 57.5 & 159.5 & 98.5 \\
\hline 9 & 49 & 79.5 & 102 & 164.2 & 123 & 56.5 & 157.5 & 99.5 \\
\hline 10 & 50 & 81 & 100.8 & 159.4 & 122 & 55 & 155.3 & 101.5 \\
\hline
\end{tabular}




\section{Conclusion}

Compared with the traditional block-matching algorithm, this new method filters the high frequency noise of the original image, and prevents the shortcoming of inaccurate block-matching on the original images; Moreover, the block-matching in the range of $\mathrm{N} \times \mathrm{N}$ on the low-frequency component image is equal to the matching search in the range of $2^{\mathrm{n}} \mathrm{N} \times 2^{\mathrm{n}} \mathrm{N}$ on the original image, the search is faster. For example, we want to make the match in the range of $\mathrm{N} \times \mathrm{N}$ on the low-frequency component image, by using the two layers wavelet decomposition, is equal to the matching search in the range of $40 \times 40$ on the original image. The calculation number of the former match is: 100(the calculation number of the Coarse matching) +18 (the calculation number of the two layers exact matching) $=118$. The calculation number of the latter match is 1600 .so the number of the new match method proposed by this paper is significantly reduced. But this algorithm is mainly for the significant moving background.

\section{Reference}

[1] Jie wei, Izidor Gerner. Foveate wavelet transform for moving target indexing[J]. SPIE .2000, 3718: 339-345

[2] Louis Shue, Ronda Venkateswarlu, Eng Thiam Lim. Tracking of low-observable Small Targets Against Clutter. SPIE. 2001, 4473: 289-295

[3] Li Xiao-hong Qian Yuan-cheng. Method of moving estimating based on wavelet field[J], Systems Engineering and Electronic Technology. 2001, 23(4), 20-22

[4] Zheng Jiang-bin, Arithmetic of Moving Targets Detection and Tracking in video frequency surveillance[J], Systems Engineering and Electronic Technology, 2002, 24(10): 34-37

[5] Jae-Soo Cho, Do-Jong Kim, Dong-Jo Park. Robust Centroid Target Tracker Based on Novel Distance Features in Cluttered Image Sequences[J]. SPIE , 2000, 4067: 1045-1055. 